\title{
Optimal power scheduling for economic dispatch using moth flame optimizer
}

\author{
N. F. Ramli ${ }^{1}$, N. A. M. Kamari ${ }^{2}$, M. A. Zulkifley ${ }^{3}$, I. Musirin ${ }^{4}$ \\ ${ }^{1,2,3}$ Department of Electrical, Electronic and System Engineering, Faculty of Engineering and Built Environment, \\ Universiti Kebangsaan Malaysia, Malaysia \\ ${ }^{4}$ Faculty of Electrical Engineering, Universiti Teknologi Mara, Malaysia
}

\begin{tabular}{l} 
Article Info \\
\hline Article history: \\
Received Jan 24, 2020 \\
Revised Mar 29, 2020 \\
Accepted Apr 14, 2020 \\
\hline Keywords: \\
Economic dispatch \\
Evolutionary programming \\
Moth flame optimizer \\
Particle swarm optimization
\end{tabular}

Article Info

optimization

\begin{abstract}
This paper proposes the optimal generator allocation to solve Economic Dispatch (ED) problem in power system using Moth Flame Optimizer (MFO). With this approach, the optimum power for each unit generating in the system will be searched based on the power constraints per unit and the amount of power demand. The objective function of this study is to minimize the total cost of generation. The amount of power loss is measured to determine the effectiveness of the proposed technique. The performance of the MFO technique is also compared to the evolutionary programming (EP) and Particle Swarm Optimization (PSO) methods. Five- and thirty-bus power system networks are selected as test systems and simulated using MATLAB. Based on simulation results, MFO provides better results in regulating the optimum power generation with minimum generation cost and power loss, compared to EP and PSO.
\end{abstract}

Copyright $(0) 2020$ Institute of Advanced Engineering and Science. All rights reserved.

\section{Corresponding Author:}

Nor Azwan Mohamed Kamari,

Department of Electrical, Electronic and System Engineering,

Faculty of Engineering and Built Environment,

Universiti Kebangsaan Malaysia,

Bangi, Selangor, Malaysia.

Email: azwank@ukm.edu.my

\section{INTRODUCTION}

Today, the issue of electricity generation has always been a major topic. The ever-increasing population, coupled with the lack of new oil sources, has led to rising fuel costs. This has forced scientists to develop a system for optimum fuel use without compromising power demand. This system will ensure proper power unit scheduling and power capacity are generated according to demand. This scheduling system can be created based on the economic dispatch (ED) calculation. The purpose of ED is to determine the optimal power generation unit with the lowest cost of power generation. At the same time, it has to produce the total amount of power supply according to the power demand and power constraints of each generator unit. ED can be categorized as one type of optimization problem. ED solutions using optimization techniques can be categorized into two parts, namely mathematical and heuristic techniques. Some of the mathematical techniques that have been introduced to solve ED problems include linear programming [1,2], quadratic programming [3] and mixed integer programming [4]. These traditional methods used to solve ED problems are time consuming and cannot solve non-linear cost functions. The solutions obtained using this method are also not optimal. Due to the disadvantages of this traditional method, heuristic approaches have been introduced as one of the solutions to ED problems. ED problems can be categorized into Convex and NonConvex. In the Non-Convex problem, the impact of the valve point is considered in the power generation cost function. Both Convex and Non-Convex problems were successfully solved using heuristic techniques as reported, respectively in $[5,6]$ and $[7,8]$. 
The artificial intelligence (AI) approach has been widely used in the field of power systems. Among the techniques used are evolutionary programming (EP) [9-12], particle swarm optimization (PSO) [13-17], ant colony optimization (ACO) [18-21], gravitational search algorithm (GSA) [22, 23] and whale opitimization algorithm (WOA) [24, 25]. EP is developed based on biological evolutionary processes. A key feature of the EP process is the mutation, in which each parent produces a new breed with different characteristics. Selection will be based on the most fittess generation. Whereas, the PSO technique attempts to mimic the behavior of a herd of animals or insects. During the search process, two types of exploration: global and local are conducted. Balance between these two explorations is the key to search for optimal solution. Meanwhile, ACO is a heuristic method that inspired by the behavior of colony of ants. This method was mainly used to solve the travelling salesman problem (TSP). Ant colonies will track the same path between the source of their food and colony, based on pheromone trail left by other ants. After finding the pheromone trail, the ants will stop the random travelling and begin to follow the trail. In this study, a new metaheuristic-based method called Moth Flame Optimizer (MFO) was introduced [26-30]. The MFO was developed based on the method of flying moths at night, called transverse orientation. At night, flying moths are guided by moonlight, where they maintain a constant angle to find their way. Among the advantages of MFO over other techniques is the simplicity and speed of the search. Its optimization capabilities have been proven and used in a variety of optimization problems such as economics delivery, engineering design and medical applications.

This study proposes efficient techniques for calculating optimal power generation capacity based on the power demand and constraints of each generator unit using MFO optimization techniques. Two events using a five-bus system with three power generation units and a thirty-bus system with six power generation were simulated using Matlab. The objective function of this optimization process is to minimize the total cost of power generation. To determine the performance of the proposed technique, the MFO approach will also be compared to the EP and PSO methods. The rest of the paper is organized as follows: Section 2 presents the formulation of ED. Section 3 explained the MFO algorithm. Section 4 discussed the optimal power scheduling algorithm. Section 5 provided the simulation results and discussions. Lastly, Section 6 presents the conclusions.

\section{FORMULATION OF ELD}

Economic load dispatch (ELD) aims to schedule power generation for each generating unit that is in accordance with the conditions and constraints of a given operation. The total production $\operatorname{cost} C_{T}$ of one power system network can be expressed as [5]:

$$
C_{T}=\sum_{i=1}^{n} C_{i}\left(P_{i}\right)
$$

where $C_{T}$ is the total production cost, $C_{i}\left(P_{i}\right)$ is the production cost of the $i^{\text {th }}$ generating unit $P_{i}$, and $n$ is number of the generating units in the system. To calculate the production cost per unit of generator, the power generation cost function $C_{i}\left(P_{i}\right)$ is based on the fuel cost coefficient and the corresponding power output for that unit. $C_{i}\left(P_{i}\right)$ is usually expressed in the form of quadratic equations as follows:

$$
C_{i}\left(P_{i}\right)=a_{i}+b_{i} \cdot P_{i}+c_{i} \cdot P_{i}^{2}
$$

Here, $a_{i}, b_{i}$, and $c_{i}$ are the fuel cost coefficients for the $i^{\text {th }}$ generating unit $P_{i}$. The objective function $J$ of the optimization process can be written as,

$$
J=\operatorname{Minimize}\left(C_{T}\right)
$$

In this study, operating limits for each generator unit and power demand are constraints that need to be considered in order to optimize the objective function. The cost function of one generator unit is unique compared to the cost function of the other generator unit. The operating limit for each generator unit can be written as,

$$
P_{i, \min } \leq P_{i} \leq P_{i, \max }
$$

where $P_{i, \max }$ and $P_{i, \min }$ are respectively the maximum and minimum operating limits of the $i^{\text {th }}$ generating unit $P_{i}$. To ensure that the power supply is enough, the amount of power generated by the generator unit must always adhere to the total power demand. A good generation system also produces a low amount of power 
loss. In this study, the amount of power generated by all generation units $P_{G}$ is equal to the sum of the total power demand $P_{D}$ and total power loss $P_{L}$.

$$
P_{G}=\sum_{i=1}^{n} P_{i}=P_{L}+P_{D}
$$

\section{MFO}

Moth-Flame Optimizer (MFO) was proposed by Mirjalili in 2015. MFO is a new optimization technique inspired by moth navigation methods in nature. Known as a transverse orientation, this approach ensures that the moth is at a constant angle to the source of light such as the moon and the candle flame for orientation [26]. In the MFO algorithm, moths are considered as search agents that move around the search space, while light sources are considered the best position now. Therefore, each agent moth will search around the source of the light to obtain a better position and update the best position. In this study, the set of moths (agent) $M$ can be described as follows:

$$
M=\left[\begin{array}{ccc}
m_{1,1} & \cdots & m_{1, d} \\
\vdots & \ddots & \vdots \\
m_{n, 1} & \cdots & m_{n, d}
\end{array}\right]
$$

Here, $d$ is the number of variable and $n$ is the number of moths. Meanwhile, the set of light source (position) $F$ can be described as follows:

$$
F=\left[\begin{array}{ccc}
F_{1,1} & \cdots & F_{1, d} \\
\vdots & \ddots & \vdots \\
F_{n, 1} & \cdots & F_{n, d}
\end{array}\right]
$$
the following:

Here, both $n$ and $d$ are the set of moth dimension. The position of $i^{\text {th }}$ moth $M_{i}$ can be described as

$$
M_{i}=S\left(M_{i}, F_{j}\right)
$$

Here, $S$ is the spiral function, $M_{i}$ is the number of $i^{\text {th }}$ moth and $F_{j}$ is the number of $j^{\text {th }}$ light source. The equation of spiral function $S$ in (5) can be shown as follows,

$$
S\left(M_{i}, F_{j}\right)=\left|M_{i}-F_{j}\right| \cdot e^{\beta \tau} \cdot \cos (2 \pi \tau)+F_{j}
$$

Here, $\beta$ is a constant and $\tau$ is the random number between -1 to 1 . The detailed explanations of the MFO algorithm process can be found in [25].

In this study, MFO techniques will be compared to existing techniques of EP and PSO. Further details on the concepts, equations and pseudocodes of EP and PSO can be found in [12] and [16], respectively.

\section{OPTIMAL POWER SCHEDULING ALGORITHM}

To find the minimum value of $C_{T}$, the optimization process for power generation scheduling for all generating units was done repeatedly, until the stopping criteria were met. Following is the optimization process for power scheduling using MFO:

a) Determine the value of $P_{i}$ using MFO based on the given constraint limit for each generating unit.

b) Calculate $C_{T}$ using (1) and (2).

c) Determine $P_{L}$ using (5).

d) Evaluate the values of the selected parameters and repeat Steps (i), (ii) and (iii) until the difference between the maximum and minimum values of objective function $J$ is 0.001 or the number of iterations reaches 100 .

\section{RESULTS AND ANALYSIS}

In this study, simulations were conducted on a five-bus system with three generators and a thirty-bus reliability test system system with six generators, respectively, as Event A and Event B. There were three cases evaluated for each event. All the events and cases carried out under the specific power demand are 
illustrated in Table 1 . Table 2 presents the fuel cost coefficients $\left(a_{i}, b_{i}\right.$, and $\left.c_{i}\right)$ with minimum and maximum power limits $\left(P_{\min }\right.$ and $\left.P_{\max }\right)$ for each generator unit in Event A and B [5].

Table 1. List of events, cases, and power demands

\begin{tabular}{|c|c|c|c|c|c|c|c|}
\hline Ever & Test System & Case & Power Demand & ht & Test System & Case & Power Demand \\
\hline \multirow{3}{*}{$\begin{array}{c}\text { Event } \\
\text { A }\end{array}$} & \multirow{3}{*}{$\begin{array}{l}\text { Five-bus system } \\
\text { with three } \\
\text { generators }\end{array}$} & Case A-1 & $75 \mathrm{M}$ & \multirow{3}{*}{$\begin{array}{c}\text { Event } \\
\text { B }\end{array}$} & \multirow{3}{*}{$\begin{array}{l}\text { Thirty-bus } \\
\text { reliability test } \\
\text { system with six } \\
\text { generators }\end{array}$} & Case B-1 & 570 \\
\hline & & Case A-2 & $135 \mathrm{MW}$ & & & Case B-2 & $790 \mathrm{MW}$ \\
\hline & & Case A-3 & 195 MW & & & Case B-3 & $1030 \mathrm{MW}$ \\
\hline
\end{tabular}

Table 2. Fuel cost coefficients and power limits for generator units in Event A and Event B

\begin{tabular}{|c|c|c|c|c|c|c|}
\hline Event & Unit & $a_{i}(\mathrm{MW})^{2}$ & $b_{i}(\mathrm{MW})$ & $c_{i}$ & $P_{\min }(\mathrm{MW})$ & $P_{\max }(\mathrm{MW})$ \\
\hline \multirow{3}{*}{ Event A } & 1 & 200 & 7.0 & 0.008 & 10 & 85 \\
\hline & 2 & 180 & 6.3 & 0.009 & 10 & 80 \\
\hline & 3 & 140 & 6.8 & 0.007 & 10 & 70 \\
\hline \multirow{6}{*}{ Event B } & 1 & 240 & 7.0 & 0.0070 & 100 & 500 \\
\hline & 2 & 200 & 10.0 & 0.0095 & 50 & 200 \\
\hline & 3 & 220 & 8.5 & 0.0090 & 80 & 300 \\
\hline & 4 & 200 & 11.0 & 0.0090 & 50 & 150 \\
\hline & 5 & 220 & 10.5 & 0.0080 & 50 & 200 \\
\hline & 26 & 190 & 12.0 & 0.0075 & 50 & 120 \\
\hline
\end{tabular}

\subsection{Event A}

In Event A, optimal power scheduling is performed on three cases: Case A-1, A-2 and A-3 with different power demands. For each case, three optimization techniques: EP, PSO and MFO are used in the power scheduling optimization process. The results for the generated power values $\left(P_{1}, P_{2}, P_{3}, P_{G}\right)$, total power loss $P_{L}$ and total power generation costs $C_{T}$ using EP, PSO and MFO optimization techniques for all cases in Event A are summarized in Table 3.

Table 3. Power scheduling for generator units in Event A

\begin{tabular}{|c|c|c|c|c|c|c|c|c|c|}
\hline Case & \multicolumn{3}{|c|}{$\mathrm{A}-1\left(P_{D}=75 \mathrm{MW}\right)$} & \multicolumn{3}{|c|}{$\mathrm{A}-2\left(P_{D}=135 \mathrm{MW}\right)$} & \multicolumn{3}{|c|}{$\mathrm{A}-3\left(P_{D}=195 \mathrm{MW}\right)$} \\
\hline Method & $\mathrm{EP}[5]$ & PSO [5] & MFO & EP [5] & PSO [5] & MFO & EP [5] & PSO [5] & MFO \\
\hline$P_{l}(\mathrm{MW})$ & 15.0071 & 11.0974 & 10.2994 & 36.2415 & 27.2484 & 27.7994 & 58.5381 & 50.0044 & 45.5813 \\
\hline$P_{2}(\mathrm{MW})$ & 21.0649 & 50.9199 & 44.5020 & 52.9243 & 63.2321 & 62.0632 & 76.8676 & 85.1790 & 79.6866 \\
\hline$P_{G}(\mathrm{MW})$ & 75.0017 & 75.0000 & 75.0000 & 135.5055 & 135.0000 & 135.0000 & 195.1007 & 195.0000 & 195.0000 \\
\hline$P_{L}(\mathrm{MW})$ & 0.0017 & 0.0000 & 0.0000 & 0.5055 & 0.0000 & 0.0000 & 0.1007 & 0.0000 & 0.0000 \\
\hline$C_{T}(\$ / \mathrm{h})$ & 1038.89 & 1032.26 & 1031.34 & 1469.25 & 1467.63 & 1467.64 & 1925.49 & 1923.76 & 1923.08 \\
\hline
\end{tabular}

For Case A-1, it is found that the values of generator units $P_{1}, P_{2}$ and $P_{3}$ selected based on EP, PSO and MFO techniques are scattered. This indicates that more options for scheduling of generating units due to the small power demand $P_{D}$. Based on the A-1 results, the MFO and PSO methods appear to generate the total amount of power $P_{G}$ that same as $P_{D}$, without power losses. This shows that both PSO and MFO perform very well compared to EP technique. In terms of power generation costs, MFO provides the lowest cost, followed by PSO and EP techniques. In Case A-2, the value of the generator unit is found to be in a small range of values, especially those selected based on PSO and MFO techniques. This is because the selected power demand is high and results in less choice for generator unit scheduling. From a $P_{D}$ standpoint, EP still produces small power loss compared to MFO and PSO techniques which give power equivalent to $P_{D}$ and zero power loss. This time, the PSO provided lower $C_{T}$ than MFO, followed by EP. The results for Case A-3 are very similar to the results of Case A-2, except for the value of $C_{T}$. The choice of generator unit values by MFO and PSO is very close to each other and there is no loss of power generated by these two techniques. The choice of generator unit value by MFO and PSO is very similar and no loss of power is generated. Like Case A-1, this time MFO managed to schedule power generation at a lower cost than PSO.

\subsection{Event B}

Same as Event A, optimal power scheduling in Event B is performed on three cases, namely Case B-1, B-2 and B-3 with different power demands using EP, PSO and MFO methods. The results for $P_{1}, P_{2}, P_{3}$, $P_{4}, P_{5}, P_{26}, P_{G}, P_{L}$ and $C_{T}$ using all three optimization techniques in Event B are summarized in Table 4. 
Table 4. Power scheduling for generator units in Event B

\begin{tabular}{|c|c|c|c|c|c|c|c|c|c|}
\hline \multirow{2}{*}{$\begin{array}{c}\text { Case } \\
\text { Method }\end{array}$} & \multicolumn{3}{|c|}{ B-1 $\left(P_{D}=570 \mathrm{MW}\right)$} & \multicolumn{3}{|c|}{$\mathrm{B}-2\left(P_{D}=790 \mathrm{MW}\right)$} & \multicolumn{3}{|c|}{$\mathrm{B}-3\left(P_{D}=1030 \mathrm{MW}\right)$} \\
\hline & EP [5] & PSO [5] & MFO & EP [5] & PSO [5] & MFO & EP [5] & PSO [5] & MFO \\
\hline$P_{l}(\mathrm{MW})$ & 246.7543 & 219.3933 & 245.4596 & 271.9857 & 326.9195 & 327.3241 & 390.9723 & 392.5252 & 399.9455 \\
\hline$P_{2}(\mathrm{MW})$ & 50.1785 & 50.0034 & 55.2024 & 63.6149 & 95.6884 & 76.9488 & 93.9187 & 145.8794 & 136.0565 \\
\hline$P_{3}(\mathrm{MW})$ & 100.7877 & 139.8063 & 116.1585 & 192.9592 & 162.7036 & 198.6866 & 208.2881 & 223.8009 & 230.9463 \\
\hline$P_{4}(\mathrm{MW})$ & 61.2489 & 50.0005 & 50.0000 & 106.7976 & 93.5241 & 54.2322 & 95.0191 & 97.9224 & 90.7998 \\
\hline$P_{5}(\mathrm{MW})$ & 51.0050 & 58.6951 & 53.1785 & 84.4445 & 60.8599 & 82.8084 & 170.7287 & 119.4907 & 121.6523 \\
\hline$P 2_{6}(\mathrm{MW})$ & 60.1072 & 52.1014 & 50.0010 & 70.2890 & 50.3045 & 50.0000 & 71.1670 & 50.3815 & 50.5996 \\
\hline$P_{G}(\mathrm{MW})$ & 570.0816 & 570.0000 & 570.0000 & 790.0909 & 790.0000 & 790.0000 & 1030.0939 & 1030.0000 & 1030.0000 \\
\hline$P_{L}(\mathrm{MW})$ & 0.0816 & 0.0000 & 0.0000 & 0.0909 & 0.0000 & 0.0000 & 0.0939 & 0.0000 & 0.0000 \\
\hline$C_{T}(\$ / \mathrm{h})$ & 6909.57 & 6892.68 & 6871.99 & 9443.24 & 9370.45 & 9347.24 & 12305.00 & 12266.20 & 12263.95 \\
\hline
\end{tabular}

The results in Event B show a pattern similar to Event A. The values for each generator unit in Case B-1 and B-2 give different values for the three optimization techniques. This is due to the low power demand, so there are many options for coupling the generator unit to get the required power demand. Meanwhile, the results for Case B-3 shows the value of each unit of the generator is in a narrow range for the three optimization techniques. This is because there are very few options for coupling generator units to obtain high power demand. Overall, the six-unit generator scheduling technique using PSO and MFO is capable of delivering the same value with power demand, without power loss. For the same generator system, EP method also produces almost identical power to the demand. However, there is a slight power loss generated by the EP, not exceeding 0.1 MW for Case B-1, Case B-2 and Case B-3. For all three cases, MFO is able to save on generating costs, followed by PSO and EP. From this result, MFO remains at the forefront of producing the lowest power generation cost and the lowest power loss compared to PSO and EP techniques.

\section{CONCLUSION}

This study proposes a power scheduling strategy using MFO to achieve optimum power output by generator units at minimum power generation costs. Two test systems which each has three different power demand is chosen as a test system and is carried out using MATLAB. The results show that MFO and PSO have successfully generated the same amount of power generation as power demand, with no loss of power. In terms of cost, MFO outperforms PSO in providing lower generation costs for the same power demand. In addition, the EP provides the highest total cost of generation compared to MFO and PSO. In fact, power generation using EP also results in power loss. In conclusion, MFO is the most appropriate technique in power scheduling for economic dispatch (ED) problems in power systems.

\section{ACKNOWLEDGEMENTS}

This study is funded by the Ministry of Education Malaysia (FRGS/1/2018/TK04/UKM/02/7).

\section{REFERENCES}

[1] A. Farag, S. Al-Baiyat and T. C. Cheng, "Economic Load Dispatch Multiobjective Optimization Procedures Using Linear Programming Techniques," IEEE Transactions on Power Systems, vol. 10, no. 2, pp. 731-738, 1995.

[2] R. A. Jabr, A. H. Coonick and B. J. Cory, "A Homogeneous Linear Programming Algorithm for The Security Constrained Economic Dispatch Problem," IEEE Transactions on Power Systems, vol. 15, no. 3, pp. 930-936, 2000.

[3] C. H. Chen and C. C. Lin, " Simple Particle Swarm Optimization for Economic Dispatch With Piecewise Quadratic Fuel Cost Sunction," in 2009 Innovative Technologies in Intelligent Systems and Industrial Applications, pp. 412-417, 2009.

[4] H. Daneshi, A. L. Choobbari, M. Shahidehpour, and Z. Li, " Mixed Integer Programming Method to Solve Security Constrained Unit Commitment With Restricted Operating Zone Limits," in 2008 IEEE International Conference on Electro/Information Technology, pp. 187-192, 2008.

[5] N. A. M. Kamari, N. A. Rahmat and I. Musirin, "Optimal Power Scheduling Strategy in Power Systems using Swarm Optimization Technique," International Journal of Advanced Trends in Computer Science and Engineering, vol. 8, issue 1.6 special issue, pp. 246-251, 2019.

[6] B. Khorramdel, A. Zare, C. Y. Chung and P. Gavriliadis, " A Generic Convex Model for a Chance-Constrained Look-Ahead Economic Dispatch Problem Incorporating an Efficient Wind Power Distribution Modeling," IEEE Transactions on Power Systems, vol. 35, no. 2, pp. 873-886, 2020.

[7] R. Dong and S. Wang, "New Optimization Algorithm Inspired by Kernel Tricks for the Economic Emission Dispatch Problem With Valve Point," IEEE Access, vol. 8, pp. 16584-16594, 2020. 
[8] M. J. Mokarram, M. Gitizadeh, T. Niknam and S. Niknam, "Robust and Effective Parallel Process to Coordinate Multi-Area Economic Dispatch (MAED) Problems in The Presence of Uncertainty," IET Generation, Transmission \& Distribution, vol. 13, no. 18, pp. 4197-4205, 2019.

[9] C. Zhu, L. Xu and E. D. Goodman, "Generalization of Pareto-Optimality for Many-Objective Evolutionary Optimization," IEEE Transactions on Evolutionary Computation, vol. 20, no. 2, pp. 299-315, 2015.

[10] A. Che, P. Wu, F. Chu and M. C. Zhou, " Improved Quantum-Inspired Evolutionary Algorithm for Large-Size Lane Reservation," IEEE Transactions on Systems, Man, and Cybernetics: Systems, vol. 45, no. 12, pp. 1535-1548, 2015.

[11] S. B. Gee, K. C. Tan and H. A. Abbass, "A Benchmark Test Suite for Dynamic Evolutionary Multiobjective Optimization," IEEE Transactions on Cybernetics, vol. 47, no. 2, pp. 461-472, 2017.

[12] N. A. M. Kamari, I. Musirin and M. M. Othman, "EP Based Optimization for Estimating Synchronizing and Damping Torque Coefficients," Australian Journal of Basic and Applied Sciences, vol. 4, no. 8, pp. 3741-3754, 2010.

[13] M. A. Hannan, M. G. M. Abdolrasol, M. Faisal, P. J. Ker, R. A. Begum and A. Hussain, "Binary Particle Swarm Optimization for Scheduling MG Integrated Virtual Power Plant Toward Energy Saving," IEEE Access, vol. 7, pp. 107937-107951, 2019.

[14] N. A. M. Kamari, I. Musirin, A. N. Dagang and M. H. M. Zaman, " PSO-Based Oscillatory Stability Assessment by Using the Torque Coefficients for SMIB," Energies, vol. 13, no. 5, pp. 1-16, 2020.

[15] M. Sato, Y. Fukuyama, T. Iizaka and T. Matsui, "Total Optimization of Energy Networks in a Smart City by MultiSwarm Differential Evolutionary Particle Swarm Optimization," IEEE Transactions on Sustainable Energy, vol. 10, no. 4, pp. 2186-2200, 2019.

[16] J. Tian, Y. Tan, J. Zeng, C. Sun and Y. Jin, "Multiobjective Infill Criterion Driven Gaussian Process-Assisted Particle Swarm Optimization of High-Dimensional Expensive Problems," IEEE Transactions on Evolutionary Computation, vol. 23, no. 3, pp. 459-472, 2019.

[17] N. A. M. Kamari, I. Musirin, Z. A. Hamid and M. H. M. Zaman, "Oscillatory Stability Prediction using PSO Based Synchronizing and Damping Torque Coefficients," Bulletin of Electrical Engineering and Informatics (BEEI), vol. 7, issue 3, pp. 331-344, 2018.

[18] Z. Yin, C. Du, J. Liu, X. Sun and Y. Zhong, "Research on Autodisturbance-Rejection Control of Induction Motors Based on an Ant Colony Optimization Algorithm," IEEE Transactions on Industrial Electronics, vol. 65, no. 4, pp. 3077-3094, 2018.

[19] X. F. Liu, Z. H. Zhan, J. D. Deng, Y. Li, T. Gu and J. Zhang, "An Energy Efficient Ant Colony System for Virtual Machine Placement in Cloud Computing," IEEE Transactions on Evolutionary Computation, vol. 22, no. 1, pp. 113-128, 2018.

[20] J. Shang, X. Wang, X. Wu, Y. Sun, Q. Ding, J. X. Liu and H. Zhang, "A Review of Ant Colony Optimization Based Methods for Detecting Epistatic Interactions," IEEE Access, vol. 7, pp. 13497-13509, 2019.

[21] Z. A. Hamid, I. Musirin, M. N. A. Rahim and N. A. M. Kamari, "Optimization Assisted Load Tracing via Hybrid Ant Colony Algorithm for Deregulated Power System," WSEAS Transactions on Power Systems, vol. 7, no. 3, pp. 145-158, 2012.

[22] S. A. Halim, A. Mohamed, N. A. M. Kamari, A. Ayob, A. H. A. Bakar and H. A. Illias," Optimisation of Zinc Oxide Surge Arrester Design using Gravitational Search Algorithm and Imperialist Competitive Algorithm," Indonesian Journal of Electrical Engineering and Computer Science (IJEECS), vol. 13, no. 3, pp. 853-860, 2019.

[23] M. K. M. Zamani, I. Musirin, M. S. Omar, S. I. Suliman, N. A. M. Ghani and N. A. M. Kamari, "Gravitational Search Algorithm Based Technique for Voltage Stability Improvement," Indonesian Journal of Electrical Engineering and Computer Science (IJEECS), vol. 9, no. 1, pp. 123-130, 2018.

[24] Q. Zhang and L. Liu, "Whale Optimization Algorithm Based on Lamarckian Learning for Global Optimization Problems," IEEE Access, vol. 7, pp. 36642-36666, 2019.

[25] N. A. M. Kamari, I. Musirin, Z. Othman and S. A. Halim, "PSS Based Angle Stability Improvement Using Whale Optimization Approach," Indonesian Journal of Electrical Engineering and Computer Science (IJEECS), vol. 8, no. 2, pp. 382-390, 2017.

[26] S. A. Halim, H. M. Rosli and H. F. Hasri, "Moth-Flame Optimization Algorithm With Different Course for Optimal Photovoltaic Location and Sizing," International Journal of Advanced Trends in Computer Science and Engineering, vol. 8, no. 1.6 special issue, pp. 145-152, 2019.

[27] S. Mirjalili, "Moth-Flame Optimization Algorithm: A Novel Nature-Inspired Heuristic Paradigm," Knowledge-Based Systems, vol. 89, pp. 228-249, 2015.

[28] C. Li, Z. Niu, Z. Song, B. Li, J. Fan and P. X. Liu, "A Double Evolutionary Learning Moth-Flame Optimization for Real-Parameter Global Optimization Problems," IEEE Access, vol. 6, pp. 76700-76727, 2018.

[29] L. Hongwei, L. Jianyong, C. Liang, B. Jingbo, S. Yangyang and L. Kai, "Chaos-Enhanced Moth-Flame Optimization Algorithm for Global Optimization," Journal of Systems Engineering and Electronics, vol. 30, no, 6, pp. 1144-1159, 2019.

[30] A. A. Elsakaan, R. A. El-Sehiemy, S. S. Kaddah and M. I. Elsaid, "Optimal Economic-Emission Power Scheduling of RERs in MGs With Uncertainty," IET Generation, Transmission \& Distribution, vol. 14, no. 1, pp. 37-52, 2020. 